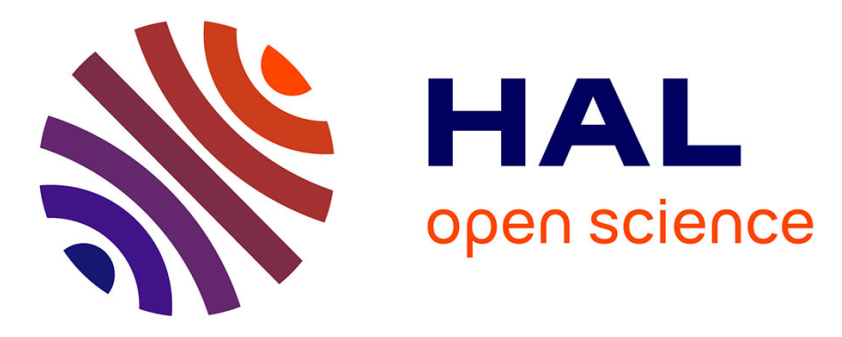

\title{
Cooperative Interference Alignment in Femtocell Networks
}

\author{
Francesco Pantisano, Mehdi Bennis, Walid Saad, Merouane Debbah
}

\section{To cite this version:}

Francesco Pantisano, Mehdi Bennis, Walid Saad, Merouane Debbah. Cooperative Interference Alignment in Femtocell Networks. IEEE GLOBECOM 2011, 2011, Texas, United States. 6 p. hal00648130

\section{HAL Id: hal-00648130 \\ https://hal-centralesupelec.archives-ouvertes.fr/hal-00648130}

Submitted on 5 Dec 2011

HAL is a multi-disciplinary open access archive for the deposit and dissemination of scientific research documents, whether they are published or not. The documents may come from teaching and research institutions in France or abroad, or from public or private research centers.
L'archive ouverte pluridisciplinaire HAL, est destinée au dépôt et à la diffusion de documents scientifiques de niveau recherche, publiés ou non, émanant des établissements d'enseignement et de recherche français ou étrangers, des laboratoires publics ou privés. 


\title{
Cooperative Interference Alignment in Femtocell Networks
}

\author{
Francesco Pantisano ${ }^{1,2}$, Mehdi Bennis ${ }^{1}$, Walid Saad ${ }^{3}$, Mérouane Debbah ${ }^{4}$ \\ ${ }^{1} \mathrm{CWC}$ - Center for Wireless Communications, Oulu, Finland, email: \{fpantisa,bennis,matla\}@ee.oulu.fi \\ ${ }^{2}$ DEIS - Dipartimento di Elettronica Informatica e Sistemistica, Bologna, Italy, email: \{francesco.pantisano\}@unibo.it \\ ${ }^{3}$ Electrical Engineering Department, Princeton University, Princeton, NJ, USA, email: saad@ princeton.edu \\ ${ }^{4}$ Alcatel-Lucent Chair in Flexible Radio, SUPÉLEC, Gif-sur-Yvette, France, email: merouane.debbah@ supelec.fr
}

\begin{abstract}
Underlay femtocells have recently emerged as a key technology that can significantly improve the coverage and performance of next-generation wireless networks. In this paper, we propose a novel approach for interference management that enables a number of femtocells to cooperate and improve their downlink rate, by sharing spectral resources and suppressing intra-tier interference using interference alignment. We formulate a coalitional game in partition form among the femtocells and propose a distributed algorithm for coalition formation. Using our approach, the femtocell access points can make individual decisions on whether to cooperate or not, while maximizing a utility function that captures the cooperative gains and the costs in terms of transmit power for information exchange. We show that, using the proposed coalition formation algorithm, the femtocells can self-organize into a network partition composed of disjoint femtocell coalitions, which constitutes the recursive core of the game. Simulation results show significant gains in terms of average payoff per femtocell, reaching up to $30 \%$ relative to the non-cooperative scheme.

Index terms: coalitional games; femtocell networks; game theory; partition form; interference alignment; recursive core.
\end{abstract}

\section{INTRODUCTION}

The deployment of femtocell access points (FAPs) is envisioned to be a key solution for providing high wireless data-rates, offloading the macrocell traffic and enhancing the coverage of existing networks (e.g., LTE or WiMAX) [1] [2]. Nevertheless, the deployment of FAPs underlaid with existing macrocell wireless networks faces several challenges, notably at the level of interference management and resource allocation. In co-channel deployments, FAPs reuse the spectral resources of the macrocell network which can lead to severe interference at both the femtocell and the macrocell tiers [3] [4]. Hence, it is of interest to propose adaptive interference management techniques, namely at the femtocell level [5].

Recently, interference alignment (IA) has been introduced as a coding technique that can achieve high multiplexing gains within interference limited environments [6], [7]. Essentially, IA is based only on linear precoding at the transmitter side and zero-forcing equalization at the receiver [7]. In [6], [8], the authors analyze the degrees of freedom resulting from using IA over different channels and for an arbitrary number of antennas per user. The authors in [6], [8] have shown that IA enables wireless users to have an interference-free communications at the cost of each user exploiting only half of the available degrees of freedom. Additional results on the achievability of the total number of degrees of freedom are presented in [9],

The authors would like to thank the Finnish funding agency for technology and innovation, Elektrobit, Nokia and Nokia Siemens Networks for supporting this work. This work has been performed in the framework of the ICT project ICT-4-248523 BeFEMTO, which is partly funded by the EU.
[10]. In essence, [9], [10] show that, by aligning all interfering signals in the same subspace from the point of view of each receiver, interference can be suppressed simply through zeroforcing equalization. In [11], an intra-cluster IA technique is applied to a clustered wireless ad hoc network to increase the probability of successful transmission. In summary, existing work in the area of interference alignment has shown that significant performance gains can be achieved by combining IA techniques with cooperative schemes in which FAPs can coordinate their transmission so as to suppress interference among each other [6], [8-10].

The main contribution of this paper is to propose a novel approach for interference management in femtocell networks in which the femtocells can cooperatively perform interference alignment so as to reduce their mutual interference, and, consequently, improve their overall performance. In the proposed approach, we study the cooperative strategies of the femtocells that enables them to form clusters inside which co-tier interference is suppressed using IA. We formulate the problem as a coalitional game in partition form in which the FAPs are the players taking autonomous decisions to create coalitions so as to maximize a utility function, which captures both the benefits from cooperation, in terms of downlink transmission rates, and the costs, in terms of transmit power for information exchange. We solve the game using the concept of a recursive core which is a key solution for coalitional games in partition form. Using simulations, we show that the proposed approach enables the FAPs to cooperate and self-organize into a stable partition while yielding significant performance gains relative to the non-cooperative scheme.

The rest of this paper is organized as follows: Section II presents the proposed system model for cooperative interference alignment. In Section III, we formulate the cooperative femtocells problem as a coalitional game and discuss its properties. In Section IV, we present the simulation results and, finally, conclusions are drawn in Section V.

Notations: In the rest of the paper, The log refers to $\log _{2}$. Bold uppercase letters (e.g., A) denote matrices, bold lowercase letters (e.g., a) denote column vectors and normal letters (e.g., a) denote scalars. The identity matrix is denoted by $\mathbf{1}$. The letter $\mathbb{E}_{\mathbf{A}}$ denotes the expectation operator over $\mathbf{A}$. Symbol $\mathbb{C}$ represent the set of complex numbers and $(\cdot)^{\dagger}$ denotes the Hermitian transpose operator.

\section{SYSTEM Model}

Consider the downlink transmission in a single macrocell network (e.g., LTE-Advanced or WiMAX) in which $N$ femtocell access points (FAPs) are deployed. These FAPs use an 
Orthogonal Frequency Division Multiple Access (OFDMA) technique over a portion of the macrocell spectrum that is reused in the femtocell tier (the remaining part of spectrum is assigned to macrocell users). Let $\mathcal{N}=\{1, \ldots, N\}$ denote the set of all FAPs. Every FAP $i \in \mathcal{N}$ serves $L_{i}$ femtocell user equipments (FUEs). Let $\mathcal{L}_{i}=\left\{1, \ldots, L_{i}\right\}$ denote the set of FUEs served by an FAP $i \in \mathcal{N}$. The FUEs belonging to the same FAP are scheduled on orthogonal subcarriers. Each transmitter (resp. receiver) is equipped with $N_{t}$ (resp. $N_{r}$ ) transmit (resp. receive) antennas. For any transmission between an FAP $i$ and one of its FUEs $k \in \mathcal{L}_{i}$, the discretetime received signal at FUE $k$, at a given time instant, is:

$$
\mathbf{y}_{k}=\sqrt{\gamma_{i k}} \mathbf{H}_{i k} \mathbf{V}_{i} \mathbf{s}_{i}+\sum_{j \in \mathcal{N}, j \neq i} \sqrt{\gamma_{j k}} \mathbf{H}_{j k} \mathbf{V}_{j} \mathbf{s}_{j}+\mathbf{n}_{k},
$$

where $\gamma_{i k}$ and $\gamma_{j k}$ denote the signal-to-noise and the interference-to-noise ratio at FAP $k \in \mathcal{L}_{i}$, respectively. $\left[\mathbf{H}_{i k}\right]$ are $N_{r} \times N_{t}$ complex matrices representing the MIMO channels between FAP $i$ and FUE $k$. $\mathbf{s}_{i} \in \mathbb{C}^{d_{i} \times 1}$ represents the $d_{i}$-dimensional signal from FAP $i$ and $\mathbf{V}_{i} \in \mathbb{C}^{N_{t} \times d_{i}}$ denotes the associated precoding matrix. $d_{i}$ represents the degrees of freedom of the transmitter-receiver pair (i.e., the multiplexing gain), for the transmitted message. Similarly, $\mathbf{s}_{j} \in \mathbb{C}^{d_{j} \times 1}$ and $\mathbf{V}_{j} \in \mathbb{C}^{N_{t} \times d_{j}}$ are the $d_{j}$-dimensional signal and the precoding matrix pertaining to interfering FAP $j$. Further, $\mathbf{n}_{k}$ represents the noise vector at receiver $k$ which is considered as a zero mean unit variance circularly symmetric additive white Gaussian noise vector (AWGN).

In existing networks, FUEs belonging to different femtocells are typically scheduled non-cooperatively, i.e., without coordination among the FAPs. Consequently, neighboring FAPs can schedule their transmissions in the downlink over the same subcarrier hence causing interference to one another and limiting the downlink performance of their FUEs. To overcome this problem and reduce the femtocell-to-femtocell interference, FAPs have an incentive to cooperate and coordinate their transmissions using advanced communication techniques such as interference alignment. In this respect, given the signal to noise and interference ratio (SINR) feedbacks from their FUEs, a group of FAPs can decide to form a coalition in which a spatial interference alignment scheme is used so as to mitigate the downlink intra-femtocell interference. In essence, the FAPs that are member of the same coalition $S \subseteq \mathcal{N}$ cooperate in order to adjust the spatial structure of their transmitted signals so as to avoid interference among themselves. Note that, even though the FAPs that are members of a given coalition $S \subseteq \mathcal{N}$ are able to mitigate interference among each other, they are still affected by non-aligned interference from FAPs (from other coalitions) in $\mathcal{N} \backslash S$. Thus, given a coalition $S$ of FAPs that are performing cooperative interference alignment, we can separate the contributions pertaining to the different types (aligned or non-aligned) of interference as follows:

$$
\begin{aligned}
\mathbf{y}_{k}=\sqrt{\gamma_{i k}} \mathbf{H}_{i k} \mathbf{V}_{i} \mathbf{s}_{i} & +\sum_{j \in S, j \neq i} \sqrt{\gamma_{j k}} \mathbf{H}_{j k} \mathbf{V}_{j} \mathbf{s}_{j} \\
& +\sum_{l \in \mathcal{N} \backslash S} \sqrt{\gamma_{l k}} \mathbf{H}_{l k} \mathbf{V}_{l} \mathbf{s}_{l}+\mathbf{n}_{k}
\end{aligned}
$$

Hence, interference alignment is achieved at an FUE $k \in$ $\mathcal{L}_{i}$, where FAP $i \in S$, if and only if there exists a zero-forcing interference suppression matrix $\mathbf{U}_{k} \in \mathbb{C}^{N_{r} \times d_{i}}$, such that:

$$
\left\{\begin{array}{c}
\mathbf{U}_{k}^{\dagger} \mathbf{H}_{j k} \mathbf{V}_{j}=0, \quad j \in S, j \neq i \\
\operatorname{rank}\left(\mathbf{U}_{k}^{\dagger} \mathbf{H}_{i k} \mathbf{V}_{i}\right)=d_{i}, \quad d_{i}>0
\end{array}\right.
$$

One must note that, by considering that the channel coefficients in $\mathbf{H}_{j k}$ are identically and independently distributed, the existence of a solution for the above IA problem solely depends on the dimensions of the problem $\left(|S|, N_{t}, N_{r}\right)$ as discussed in [12]. For instance, when the target multiplexing gain $d_{i}$ at the FUE is equal to one, a solution to the system (3) exists for the interference channel composed by $|S|-1$ interfering transmissions plus the useful signal if and only if $N_{t}+N_{r} \geq|S|$.

Moreover, to find the precoding and interference suppression matrices one can use existing iterative algorithms such as in [13, Algorithm 1], [12]. Therefore, the interference from members of the same coalition can be suppressed, yielding, after projection, the following signal at receiver $k$ :

$$
\overline{\mathbf{y}}_{k}=\sqrt{\gamma_{i k}} \mathbf{U}_{k}^{\dagger} \mathbf{H}_{i k} \mathbf{V}_{i} \mathbf{s}_{i}+\sum_{l \in \mathcal{N} \backslash S} \sqrt{\gamma_{l k}} \mathbf{U}_{k}^{\dagger} \mathbf{H}_{l k} \mathbf{V}_{l} \mathbf{s}_{l}+\mathbf{U}_{k}^{\dagger} \mathbf{n}_{k},
$$

where the remaining interference term is due to non-aligned transmissions in the coalitions formed outside $S$, i.e., in $\mathcal{N} \backslash S$.

For ease of analysis, we assume that the signals $\mathbf{s}_{l}$ are not known at the receiver and that Gaussian code books are used, which is a common assumption in the literature [14]. As a result, the interference Gaussian. Note that, even when the aggregate noise is not Gaussian, this assumption remains reasonable since it constitutes a lower bound on the actual capacity. Further, we focus on the contribution of the interference-plus-noise as in (4), and analyze an expression of the achievable rate assuming complete knowledge of the channel conditions. In this respect, we utilize the mutual information between the transmitted and received signal given, for an FAP $i \in \mathcal{N}$ as follows:

$$
\begin{aligned}
I\left(\mathbf{s}_{i}, \overline{\mathbf{y}}_{k}, \pi_{N}\right) & =h\left(\overline{\mathbf{y}}_{k}, \pi_{N}\right)-h\left(\overline{\mathbf{y}}_{k}, \mathbf{s}_{i}, \pi_{N}\right) \\
& =\log \operatorname{det}\left(\pi e \mathbf{Q}_{\bar{y}_{k}}\right)-\log \operatorname{det}\left(\pi e \mathbf{Q}_{\bar{t}_{k}}\right),
\end{aligned}
$$

where we let $\overline{\mathbf{t}}_{k}$ denote the interference-plus-noise in (4) (i.e., the last two summands), while $\mathbf{Q}_{\bar{y}_{k}}$ and $\mathbf{Q}_{\bar{t}_{k}}$ represent the covariance matrices of the received signal and the received interference-plus-noise, respectively. In particular, if we denote $\tilde{\mathbf{H}}_{l k}=\sqrt{\gamma_{l k}} \mathbf{U}_{k}^{\dagger} \mathbf{H}_{l k} \mathbf{V}_{l}, \forall(l \in \mathcal{N} \backslash S)$, the covariance matrices can be expressed by:

$$
\begin{gathered}
\mathbf{Q}_{\bar{t}_{k}}=\mathbb{E}_{\mathbf{s}_{l}, \mathbf{n}_{k}}\left[\mathbf{t}_{k}, \mathbf{t}_{k}^{\dagger}\right]=\sum_{l \in \mathcal{N} \backslash S} \tilde{\mathbf{H}}_{l k} \mathbf{Q}_{l} \tilde{\mathbf{H}}_{l k}^{\dagger}+\sigma_{n}^{2} \mathbf{1} \\
\mathbf{Q}_{\bar{y}_{k}}=\mathbb{E}_{\mathbf{s}_{i}, \mathbf{s}_{l}, \mathbf{n}_{k}}\left[\overline{\mathbf{y}}_{k}, \overline{\mathbf{y}}_{k}^{\dagger}\right]=\tilde{\mathbf{H}}_{i k} \mathbf{Q}_{i} \tilde{\mathbf{H}}_{i k}^{\dagger}+\mathbf{Q}_{\bar{t}_{k}},
\end{gathered}
$$

where $\mathbf{Q}_{i}$ represents the covariance matrix of the signal $\mathbf{s}_{i}$. Moreover, we use the fact that, for the received noise $\mathbf{n}_{k}$, $\mathbb{E}_{\mathbf{n}_{k}}\left[\mathbf{n}_{k}, \mathbf{n}_{k}^{\dagger}\right]=\sigma_{n}^{2} \mathbf{1}$. Note that, $\mathbf{Q}_{\bar{t}_{k}}$ is present in the expression of $\mathbf{Q}_{\bar{y}_{k}}$. In addition, in order to compute the achievable rate, we use that $\log \operatorname{det}(\pi e(\mathbf{A}+\mathbf{B}))-\log \operatorname{det}(\pi e(\mathbf{B}))=$ $\log \operatorname{det}\left(\mathbf{1}+\mathbf{A} \mathbf{B}^{-1}\right)$ if $\mathbf{B}$ is invertible. Since we consider an interference-limited scenario, we neglected the noise term in 
(6). The average achievable rate at receiver $k$ in coalition $S \subseteq \mathcal{N}:$

$$
\begin{aligned}
R_{i k}\left(\mathbf{s}_{i}, \overline{\mathbf{y}}_{k}\right)= & \mathbb{E}_{\mathbf{H}}\left\{\left[\operatorname { l o g } \operatorname { d e t } \left(\mathbf{1}+\tilde{\mathbf{H}}_{i k} \mathbf{Q}_{i} \tilde{\mathbf{H}}_{i k}^{\dagger} .\right.\right.\right. \\
& \left.\left.\left.\cdot\left(\sum_{l \in \mathcal{N} \backslash S} \tilde{\mathbf{H}}_{l k} \mathbf{Q}_{l} \tilde{\mathbf{H}}_{l k}^{\dagger}\right)^{-1}\right)\right]\right\}
\end{aligned}
$$

For performing cooperative IA, the FAPs belonging to the same coalition need to exchange information which, in turn, incurs a cost for cooperation. In this context, we consider a cost in terms of the transmit power that each FAP spends to send its information to the other coalition member. This information exchange phase occurs via a wireless broadcast transmission over a control channel such as the X2 interface [5]. Given the broadcast nature of the wireless channel, we consider that each FAP $i \in S$ exchanges its information to the other coalition member by transmitting its data to the farthest cooperating FAP in the coalition. Hence, given a coalition $S$, the power needed to exchange information between an FAP $i \in S$ and the farthest cooperating FAP $\hat{i} \in S$ is:

$$
\bar{P}_{i}(\hat{i})=\frac{\nu_{0} \cdot \sigma_{n}^{2}}{\left|H_{i \hat{i}}\right|^{2}}
$$

where $\nu_{0}$ is the minimum SNR required at the potential coalition partner $\hat{i}$, and $\left|H_{i, \hat{i}}\right|^{2}$ represents the channel gain between FAPs $\hat{i}$ and $i$, over the common control channel. Further, FAPs need to satisfy the power constraint $P_{i}=$ $\mathbf{Q}_{s_{i}}=\mathbb{E}\left[\mathbf{s}_{i}^{\dagger} \mathbf{s}_{i}\right] \leq P_{\max }{ }^{1}$, while still accounting for the pilot transmit penalty. The constraint on the total transmit power thus becomes:

$$
P_{i}+\bar{P}_{i}(\hat{i}) \leq P_{\max }
$$

Clearly, the cost defined in (8) depends on the spatial distribution of the FAPs in the network and on the minimum SINR required. We consider that the FAPs constraint in (9) also stands for the transmission during information exchange (e.g., over the X2 interface). Without loss of generality, the coalition formation framework presented in this article can also be applied to different cost functions ${ }^{2}$.

\section{Femtocell Cooperation as a Coalitional Game \\ A. Coalitional Game Concepts}

In order to mathematically model the femtocell cooperation problem, we formulate a coalitional game between the FAPs. Due to co-channel interference, the rate achieved by the FAPs members of any coalition $S$ that forms in the network is affected by the cooperative behavior of the FAPs outside $S$, i.e., the FAPs in $\mathcal{N} \backslash S$. In other words, the performance of a coalition depends on the partition of the network $\pi_{\mathcal{N}}\left(\pi_{\mathcal{N}}\right.$ is a partition of $\mathcal{N}$ ) to which it belongs. Given a partition $\pi_{\mathcal{N}}$ of $\mathcal{N}$, the total rate that any coalition of FAPs $S \in \pi_{\mathcal{N}}$ achieves, depends on the external interference that is generated by the coalitions of FAPs in $\mathcal{N} \backslash S$. Hence, given this property,

\footnotetext{
${ }^{1}$ Clearly, the same constraint applies to the covariances of the interferers $j$, and it is still denoted with subscript $j$ instead of $i$.

${ }^{2}$ For example, since femtocells are connected to each other through the X2 interface, the proposed cost can be replaced by a cost for synchronization or for trading additional information such as bandwidth usage or priority policies.
}

one suitable framework for modeling the femtocell cooperation problem is that of a coalitional game in partition form with transferable utility (TU) defined as follows [15]:

Definition 1: A coalitional game in partition form with transferable utility (TU) is defined by a pair $(\mathcal{N}, v)$ where $\mathcal{N}$ is the set of players, and $v$ is a value function that assigns, for every partition $\pi_{\mathcal{N}}$ and every coalition $S \subseteq \mathcal{N}, S \in \pi_{\mathcal{N}}$, a real number that represents the total utility (benefit) that players in $S$ can achieve.

Given the set of FAPs $\mathcal{N}$, our next step is to define a suitable value function $v\left(S, \pi_{\mathcal{N}}\right)$ that reflects the total benefit that coalition $S$ achieves when acting within partition $\pi_{\mathcal{N}}$. For any coalition $S$, each FAP $i \in S$ obtains a certain payment from its served FUEs that is proportional to the amount of rate offered to these FUEs. Hence, any FAP $i \in S$ charges a certain price $\alpha_{i k}$ per every unit rate offered to a given FUE $k \in \mathcal{L}_{i}$. As a result, given a partition $\pi_{\mathcal{N}}$, for any coalition $S$, the value function $v\left(S, \pi_{\mathcal{N}}\right)$ can be defined as the total achieved revenue as follows:

$$
\mathrm{v}\left(\mathrm{S}, \pi_{\mathrm{N}}\right)=\sum_{i=1}^{|S|} \sum_{k=1}^{L_{i}} \alpha_{i k} R_{i k}\left(\mathbf{s}_{i}, \overline{\mathbf{y}}_{i}, \pi_{\mathcal{N}}\right)
$$

where $R_{i k}\left(\mathbf{s}_{i}, \overline{\mathbf{y}}_{i}, \pi_{\mathcal{N}}\right)$ is the rate achieved by FUE $k$ served by FAP $i \in S$ and $\alpha_{i k}$ is the price per unit rate that FAP $i \in S$ charges to FUE $k$. Note that, the rate $R_{i k}\left(\mathbf{s}_{i}, \overline{\mathbf{y}}_{i}, \pi_{\mathcal{N}}\right)$ is function of the interference generated, not only by the FAPs inside $S$ but also by the FAPs in $\mathcal{N} \backslash S$, and, thus, depends on the partition $\pi_{\mathcal{N}}$ that takes place in the network. The dependence of the rate on the actual partition implies that, for a coalition $S,(10)$ is a function of the coalitions that the FAPs in $\mathcal{N} \backslash S$ form and, thus, the game is in partition form.

The value in (10) represents the total revenue that a coalition $S$ obtains by acting cooperatively controlled by a pricing factor $\alpha_{i k}$. For example, this revenue can represent payments that the FUEs make to their serving FAPs. Clearly, the revenue is a transferable quantity that can be apportioned in any way between the coalitional members. Hence, using (10), we clearly have a game with TU and our next step is to propose a fair scheme for dividing the revenue of a coalition $S$ between its members. In fact, for TU games, beyond the total revenue that a coalition $S$ achieves as per (10), one has to also characterize the payoff $x_{i}$ that each player (i.e., FAP) in the coalition $S$ receives, i.e., the benefit of every member in the coalition. For this purpose, without loss of generality, we adopt an egalitarian fair payoff division method using which the extra value given by the coalition formation is equally divided among the players in the coalition. Thus, the payoff for FAP $i$ in any coalition $S$ can be found from (10) by an egalitarian fair division, as follows:

$x_{i}\left(S, \pi_{\mathcal{N}}\right)=\frac{1}{|S|}\left(v\left(S, \pi_{\mathcal{N}}\right)-\sum_{j \in S} v\left(\{j\}, \pi_{\mathcal{N}}\right)\right)+v\left(\{i\}, \pi_{\mathcal{N}}\right)$.

Note that the payoff is zero if the power used for information exchange in the coalition exceeds the limit given by (9). We also note that the egalitarian fair distribution does not imply dividing the whole utility equally but rather the extra 
benefits (relative to the non-cooperative case) equally, while conserving individual rationality, i.e., ensuring that an FAP that is member of $S$ does not get less benefit than when acting non-cooperatively.

Furthermore, given two payoff vectors $\boldsymbol{x}, \boldsymbol{y} \in \mathbb{R}^{N}$, we write $\boldsymbol{x}>_{S} \boldsymbol{y}$ if $x_{i} \geq y_{i}$ for all $i \in S \subset \mathcal{N}$ and for at least one $j \in S$ $x_{j}>y_{j}$. We also define an outcome as couple $\left(\mathbf{x}, \pi_{\mathcal{N}}\right)$, where $\mathbf{x}$ is a payoff vector resulting from a partition $\pi_{\mathcal{N}}$. Finally, let $\Omega(\mathcal{N}, v)$ denote the set of all the possible outcomes of $\mathcal{N}$.

Given these definitions, we have a coalitional game in partition form $(\mathcal{N}, v)$ between the FAPs and our objective is to propose a solution that allows the FAPs to self-organize and form coalitions while increasing their revenue (i.e., their total rate) given the costs for cooperation as in (10).

\section{B. Recursive core}

In order to solve the proposed femtocell coalition formation game in partition form, we use the concept of a recursive core introduced and discussed in [16]. The recursive core is a key solution concept for coalitional games that have dependence on externalities, i.e., in partition form. In essence, the recursive core draws a parallel with the well-known core concept of games in characteristic form [16]. The recursive core is a suitable outcome of a coalition formation process that accounts for externalities across coalitions, which, in the considered game, are represented by the mutual interference between coalitions of FAPs. To define the recursive core we need to first introduce the concept of a residual game:

Definition 2: A residual game $(\mathcal{R}, v)$ is a coalitional game in partition form defined on a set of players $\mathcal{R}$, after the players in $\mathcal{N} \backslash \mathcal{R}$ have already organized themselves in a certain partition. Players outside $\mathcal{R}$ are called deviators, while the players in $\mathcal{R}$ are called residuals.

Consider a coalitional game $(\mathcal{N}, v)$ and let $S$ be a certain coalition of deviators. Then, let $\mathcal{R}=\mathcal{N} \backslash S$ denote the set of residual players. The residual game $(\mathcal{R}, v)$ is defined as a game in partition form over the set $\mathcal{R}$. Clearly, a residual game is still in partition form and it can be solved as an independent game, regardless of how it was generated as discussed in [16]. To better present this concept, we will provide an intuitive introduction. For instance, when some deviators reject an existing partition and decide to reorganize themselves into a different partition, their decisions will, in general, affect the payoff of the residual players. As a result, the residual players form a new game that is part of the original game (e.g., the game over the whole set $\mathcal{N}$ ), but with a certain part of the partition (composed by deviators) already fixed. In consequence, one of the main attractive properties of a residual game is its consistency as well as the possibility of dividing any coalitional game in partition form into a number of residual games which, in essence, are easier to solve. In fact, any game in partition form can be seen as a collection of residual games, each one of which can be solved as if it was the original one. The solution of a residual game is known as the residual core which is defined as follows:

Definition 3: The residual core of a residual game $(\mathcal{R}, v)$ is a set of possible game outcomes, i.e., partitions of $\mathcal{R}$ that can be formed.

One can see that given any coalitional game $(\mathcal{N}, v)$, residual games are smaller than the original one and therefore computationally easier to analyze. Given any coalitional game $(\mathcal{N}, v)$, the recursive core solution can be found by recursively playing residual games, which, in fact, yields the following definition as per [16, Definition 2]:

Definition 4: The recursive core of a coalitional game $(\mathcal{N}, v)$ is inductively defined in four main steps:

1) Trivial Partition. Let $(\mathcal{N}, v)$ be a coalitional game. The recursive core of a coalitional game where $\mathcal{N}=\{i\}$ is composed by the only outcome with the trivial partition composed by the single player $i: C(\{i\}, v)=(v(i), i)$.

2) Inductive Assumption. Proceeding recursively, suppose the recursive core $C(\mathcal{R}, v)$ for each game with at most $N-1$ players has been defined. Now, we define the assumption $A(\mathcal{R}, v)$ about the game $(\mathcal{R}, v)$ as follows: $A(\mathcal{R}, v)=C(\mathcal{R}, v)$, if $C(\mathcal{R}, v) \neq 0 ; A(\mathcal{R}, v)=$ $\Omega(\mathcal{R}, v)$, otherwise.

3) Dominance. An outcome $\left(\mathbf{x}, \pi_{\mathcal{N}}\right)$ is dominated via a coalition $S$ if for at least one $\left(\mathbf{y}_{\mathcal{N} \backslash S}, \pi_{\mathcal{N} \backslash S}\right) \in A(\mathcal{N} \backslash S, v)$ there exists an outcome $\left(\left(\mathbf{y}_{S}, \mathbf{y}_{\mathcal{N} \backslash S}\right), \pi_{S} \cup \pi_{\mathcal{N} \backslash S}\right) \in$ $\Omega(\mathcal{N}, v)$ such that $\left(\mathbf{y}_{S}, \mathbf{y}_{\mathcal{N} \backslash S}\right)>_{S} \mathbf{x}$.

4) Core Generation. The recursive core of a game of $|\mathcal{N}|$ players is the set of undominated outcomes and we denote it by $C(\mathcal{N}, v)$.

Note that, in Definition 4, the concept of dominance in step 3 ) inherently captures the fact that the value of a coalition depends on the belonging partition. Hence, it can be expressed in the following way: given a current partition $\pi_{\mathcal{N}}$ and the respective payoff vector $\mathbf{x}$, an undominated coalition $S$ represents a deviation from $\pi_{\mathcal{N}}$ in such a way that the resulting outcome $\left(\left(\mathbf{y}_{S}, \mathbf{y}_{\mathcal{N} \backslash S}\right), \pi_{S} \cup \pi_{\mathcal{N} \backslash S}\right)$ is more rewarding for the players of $S$, compared to $\mathbf{x}$.

Since a partition uniquely determines the payoffs of all the players in the game, the recursive core can be seen a set of partitions that allow the players to organize in a way that provides them with the highest payoff. It is worth stressing that the recursive core verifies the properties of rationality, welldefinition and efficiency [16]. In detail, with rationality it is intended that players never choose an inferior (i.e., dominated) strategy, therefore, they always pursue a profitable strategy. The recursive core is also well-defined because when it exists, its solution is unique. Furthermore, efficiency is a consequence of the fact that there is no preference in the set composed by the recursive core, and thus, all the included partitions are equivalent in terms of individual payoff. Moreover, In [16, Lemma 10], the author proves that the term core is justified, since the recursive core is, as previously mentioned, a natural generalization of the core in characteristic form, to games with externalities. Consequently, when applied to a game in characteristic function form, the recursive core coincides with the core, as classically defined [17].

Given these properties, once a partition in the recursive core takes place, the players have no incentive to abandon it, because any deviation would be detrimental. As a result, 


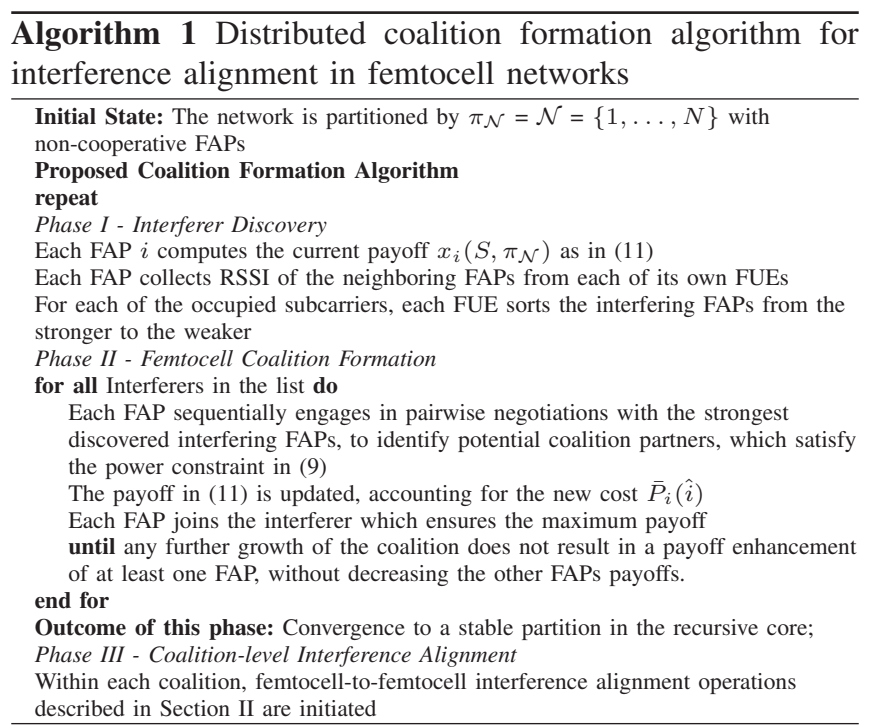

a partition in the recursive core is also stable since it is a partition which ensures the highest possible payoff for each one of the players who have no incentive to leave this partition.

In [16], the author shows that the existence of the recursive core requires at least one residual core (and not all of them) to be nonempty. This means that at least a subset of the players in the network must have defined a preference on how to organize themselves, i.e., how to partition the network. In detail, an empty residual core reflects a case in which the players of the corresponding residual game do not identify any preferred network partition, or in our proposed FAP cooperation scenario, can equivalently choose between cooperating or not.

Therefore, for the proposed FAPs coalitional game, the emptiness of a residual core does not happen and this can be justified as follows. As per Definition 4, the recursive core is evaluated through a sequence of residual games over subsets of players (i.e., FAPs in our case) in the network. When a given residual core is empty, it is still possible to solve a larger game which contains it, as a residual game, in a nested fashion. Hence, the existence of the recursive core is in fact guaranteed as long as one can find at least one residual core that is nonempty. Thus, the recursive core is a solution concept that exists for any game in partition form, unless all the residual cores are empty. This latter case is very unlikely since it would represent a network in which FAPs are indifferent (i.e., have the same payoff) between states in which they are actually suppressing interference (e.g., cooperatively using interference alignment).

In a nutshell, for the proposed FAPs coalitional game, one can use the concept of residual cores in order to find a partition in the recursive core, i.e., a stable and efficient partition, as will be further described in the next section. To reach a partition in the recursive core, the FAPs can use Algorithm 1.

\section{Simulation Results}

For system-level simulations, we consider a single hexagonal macrocell with a radius of $500 \mathrm{~m}$ within which $N$ FAPs are randomly deployed. Each FAP $i \in \mathcal{N}$ serves $L_{i}=2$ FUEs

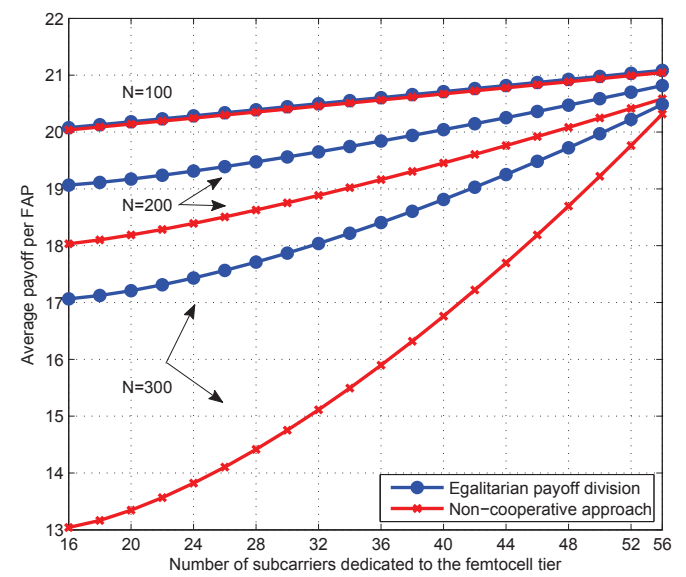

Fig. 1. Average individual user payoff as a function of the number of subcarriers dedicated to the femtocell tier for different number of FAPs.

scheduled over $L_{i}$ orthogonal subcarriers which are typical values as in [5]. FAPs and FUEs are equipped with $N_{t}=N_{r}=$ 2 antennas. An open access policy is adopted at each FAP. We set each FAP's maximum transmit power to $P_{\max }=10 \mathrm{dBm}$, which includes both the power for data transmission and the cost for cooperation in (8). Transmissions are affected by distance dependent path loss shadowing according to the 3GPP specifications [18]. Moreover, a wall loss attenuation of $12 \mathrm{~dB}$ affects femtocell-to-femtocell transmissions. The considered macrocell has 110 available subcarriers, each one having a bandwidth of $180 \mathrm{KHz}$, and dedicates 16 OFDMA subcarriers to femtocell transmissions. For both femto users and MUEs, we assume that power control fully compensates for the path loss. The signal-to-noise ratio required for information exchange is $\nu_{0}=5 \mathrm{~dB}$. The parameters $\alpha_{i k}$ are set to 1 by simulation choice. To leverage channel variations, statistical results are averaged on 10000 simulation rounds.

Figure 1 depicts the average individual FAP payoff as a function of the number of subcarriers dedicated to the femtocell tier, for $N=\{100,200,300\}$. This figure shows that the ratio between number of FAPs and number of dedicated subcarriers plays a key role, as interference becomes critical over a high congested spectrum. For all of the considered network sizes, the cooperative and non-cooperative strategies lead to similar payoffs when a large portion of spectrum is available, due to the low density of interferers for a given subcarrier. Conversely, the benefit from coalition formation becomes relevant in a high congested spectrum and, in the case of $N=300$ allows for a maximum spectrum saving of $38 \%$ with respect to the non-cooperative case. In addition, Figure 1 demonstrates that the proposed coalitional game model has a significant advantage over the non-cooperative case, increasing with $N$ and resulting in an improvement of up to $30 \%$ for $N=300$ and 16 subcarriers.

In Fig. 2, we show the average and the average maximum size of the FAP coalitions in the recursive core for a target SNR for information exchange of $\nu_{0}=5 \mathrm{~dB}$. Fig. 2 shows that, for small networks, the FAPs have low incentive to cooperate, and, thus, the recursive core is mainly populated by singleton 


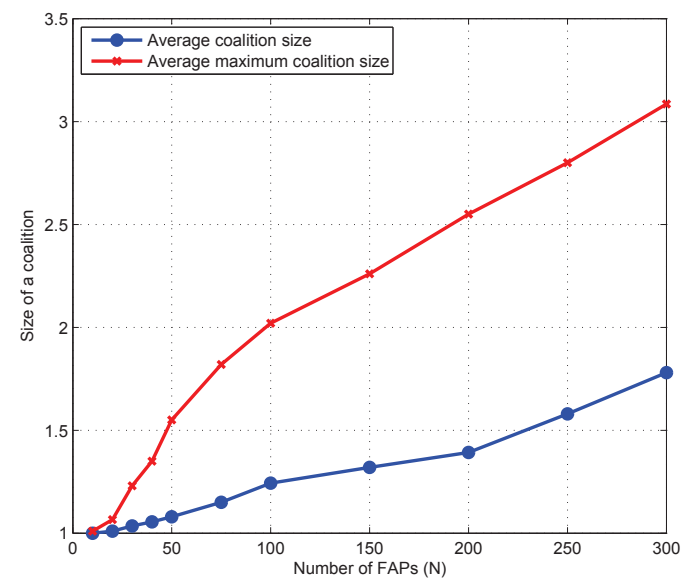

Fig. 2. Average and average maximum coalition size as function of the number of FAPs.

coalitions. In contrast, as $N$ grows, the density of FAPs and the interference level increases, thus, a cooperative strategy becomes more rewarding, as seen through the increase in the average size of the coalitions. As a matter of fact, by forming coalitions, nearby interfering FAPs can still utilize the same subcarriers and suppress interference via alignment. However, the maximum size of a coalition is limited by the cost for cooperation as well as the inherent efficiency of IA.

Figure 3 shows the growth of the number of coalitions, i.e., the size of a partition in the recursive core, while the number of FAPs increases. Additionally, the average number of iteration in the proposed algorithm is observed. The network is initially organized in a non-cooperative structure where each FAP represents a singleton coalition, therefore the number of coalitions equals the number of FAPs (grey dotted line in Figure 3) and, since interferers are out of range of cooperation, the number of iterations is minimum. Initially, for $N<50$ cooperation seldom occurs, due to the large distance between mutual interferers. As $N$ increases $(50<N<300)$, the network topology changes with the emergence of new coalitions. The number of iterations depend on the number of potential coalitional partners which satisfy the constraint in (9). Therefore, Figure 2 and Figure 3 show that the incentive towards cooperation becomes significant when the femtocells' spectrum becomes more congested and femtocells are densely deployed in the network.

\section{Conclusions}

In this paper, we have presented a cooperative framework aimed at interference mitigation in a femtocell network. We have formulated the problem as a coalitional game in partition form and proposed a distributed coalition formation algorithm that enables FAPs to decide on whether to cooperate or not, while accounting for the cost in terms of power for information exchange. We have shown that the proposed algorithm reaches a stable partition, lying in the recursive core of the studied game. Within every formed coalition, we have adopted an interference mitigation technique based on the alignment of interfering signals from members of the same coalition. Results have shown that the femtocell performance is critically limited by the interference, therefore, the proposed cooperative

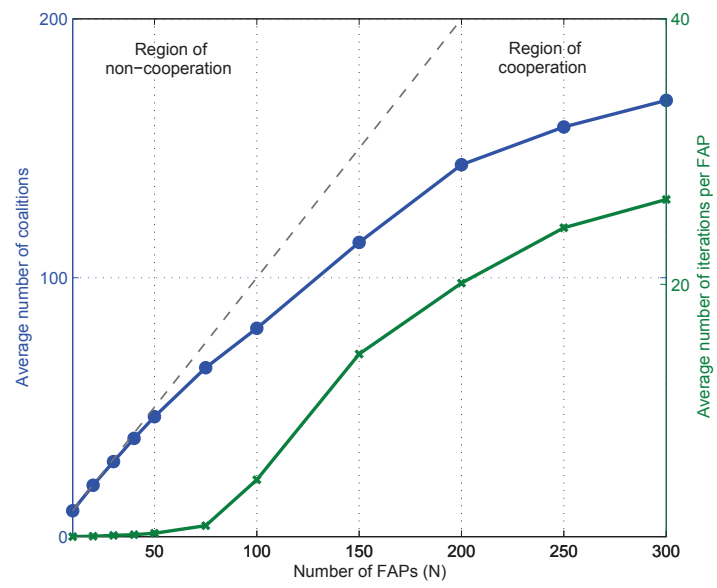

Fig. 3. Average number of coalitions and average number of iterations per FAP until convergence to a stable partition in the recursive core.

strategy among interfering femtocells can provide significant gains, in terms of average payoff per femtocell, reaching up to $30 \%$ relative to the non-cooperative case.

\section{REFERENCES}

[1] I. T. S. Sesia and M. Baker, LTE - the UMTS long term evolution. A John Wiley and Son publication - UK, Aug. 2009.

[2] 3GPP, "Requirements for further advancements for E-UTRA (LTEAdvanced),"3GPP Technical report (TR 36.913) v8.0.1, Mar. 2009.

[3] D. Lopez-Perez, A. Valcarce, G. de la Roche, and J. Zhang, "OFDMA femtocells: A roadmap on interference avoidance," IEEE Communications Magazine, vol. 47, no. 9, pp. 41-48, 2009.

[4] V. Chandrasekhar and J. G. Andrews, "Spectrum allocation in tiered cellular networks," IEEE Transactions on Communications, vol. 57, no. 10, pp. 3059-3068, October 2009.

[5] 3GPP, "Service requirements for Home Node B (HNB) and Home eNode B (HeNB)," 3GPP Technical Specification (TS 22.200) v9.5.0, Oct. 2010.

[6] S. Jafar and S. Shamai, "Degrees of freedom region of the MIMO X channel," IEEE Transactions on Information Theory, , vol. 54, no. 1, pp. $151-170,2008$.

[7] C. Suh, M. Ho, and D. Tse, "Downlink interference alignment," vol. abs/1003.3707, May 2010. [Online]. Available: http://arxiv.org/abs/ 1003.3707

[8] V. Cadambe and S. Jafar, "Interference alignment and the degrees of freedom of wireless X networks," IEEE Transactions on Information Theory, vol. 55, no. 9, pp. 3893 -3908, 2009.

[9] T. Gou and S. Jafar, "Degrees of freedom of the K user MIMO interference channel," in 42nd Asilomar Conference on Signals, Systems and Computers, 2008, pp. $126-130$.

[10] S. W. Peters and R. Heath, "Cooperative algorithms for MIMO interference channels," CoRR, vol. abs/1002.0424, 2010. [Online]. Available: http://arxiv.org/abs/1002.0424

[11] R.Tresch and M. Guillaud, "Performance of interference alignment in clustered wireless ad hoc networks," in IEEE International Symposium on Information Theory (ISIT), Austin, TX, USA, 2010.

[12] R. Tresch, M. Guillaud, and E. Riegler, "On the achievability of interference alignment in the k user constant mimo interference channel," in IEEE/SP 15th Workshop on Statistical Signal Processing, 2009, pp. $277-280$.

[13] K. Gomadam, V. Cadambe, and S. Jafar, "Approaching the capacity of wireless networks through distributed interference alignment," in IEEE Global Telecommunications Conference,(IEEE GLOBECOM 2008), 30 2008.

[14] S. Mathur, L. Sankar, and N. Mandayam, "Coalitions in cooperative wireless networks," IEEE Journal on Selected Areas in Communications, vol. 26, no. 7, pp. 1104-1115, 2008.

[15] W. Saad, Z. Han, and A. Hjørungnes, "A distributed coalition formation framework for fair user cooperation in wireless networks," Wireless Communications, IEEE Transactions on, vol. 8, no. 9, pp. 4580-4593, 2009

[16] L. Kóczy, "A recursive core for partition function form games," no. 031, 2007. [Online]. Available: http://econpapers.repec.org/RePEc:dgr: umamet:2006031

[17] D. Ray, A Game-Theoretic Perspective on Coalition Formation. New York, USA: Oxford University Press.

[18] 3GPP, "Physical layer aspects for evolved UTRA (Release 7)," 3GPP Technical report (TR 25.814) v7.1.0, Oct. 2006. 